\title{
Effects of an atelocollagen sponge during the wound healing of tooth extraction sockets at an early stage
}

\author{
Shoichiro Iwata ${ }^{1}$, Kenichi Matsuzaka ${ }^{1,2}$, Takashi Inoue ${ }^{1,2}$ \\ ${ }^{\prime}$ Department of Clinical Pathophysiology, Tokyo Dental College, Chiba, Japan \\ ${ }^{2}$ Oral Health Science Center hrc7, Tokyo Dental College, Chiba, Japan
}

Abstract: The purpose of this study was to evaluate the morphological and molecular effects of atelocollagen sponges placed in rat tooth extraction sockets in their early stages of wound healing. Right incisors of rats were extracted, and atelocollagen sponges were placed into the tooth extraction sockets. Sockets without atelocollagen served as controls. Extraction socket tissues were histologically evaluated for 3 weeks after surgery, and mRNA expression levels for type I collagen and osteocalcin were also quantified. At the time that non-specific fibrous connective tissue invaded the alveolar bone ridge area of the sockets from the outside in the control group, new bone formation was seen up to the level of the alveolar bone ridge in the experimental group. There was a significantly higher expression level for type I collagen mRNA in the experimental group, whereas there was no significant difference in the expression of osteocalcin mRNA between the two groups. These results suggest that atelocollagen plays a role in maintaining space by stimulating collagen production but does not accelerate cell proliferation or osteoinduction.

[Oral Med Pathol 2010; 15: 15-20 doi: 10.3353/omp.15.15]

Key words: atelocollagen, tooth extraction socket, morphometric analysis, Ki-67, type I collagen mRNA, osteocalcin mRNA, rat

Correspondence: Kenichi Matsuzaka, Oral Health Science Center hrc7, Department of Clinical Pathophysiology, Tokyo Dental College, 1-2-2 Masago, Mihama-ku, Chiba 261-8502 Japan

Phone: +81-43-270-3581, Fax: +81-43-270-3583, E-mail: matsuzak@tdc.ac.jp

\section{Introduction}

Recent advances in bioengineering have introduced materials that enhance wound healing (1-2). A decrease in alveolar bone, a phenomenon that occurs in early stages after tooth extraction, is challenge for prosthodontic treatment and dental implant therapy. Because bone augmentation in the absorbed alveolar bone increases the patient burden and has a risk for bacterial infection, maintaining of alveolar bone shape just after tooth extraction plays a convenient role for dental implant therapy. Many kinds of artificial or biomaterials, such as atelocollagen, beta-TCP, hydroxylapatite, burned bone tissue from animals, and so on, are used in tooth extraction sockets. Other materials used in tooth extraction sockets include micro fibrous collagen, cellulose and gelatin sponge. These materials help maintain the hemostasis and are a barrier to stimulation from outside, but are associated with delayed wound healing. Although collagen is the most abundant protein in the human body, the cross-linked $\mathrm{N}$-telopeptide is an antigenic moiety existing at the terminus of type I collagen. Atelocollagen is type I collagen after removal of the N-telopeptide, which has been reported to have minimal immunogenicity (3).

There have been many reports about the effects of atelocollagen (1-2, 4-8), but only a few studies have comprehensively examined it using morphological, mathematical and/or biochemical analyses. The purpose of this study was to evaluate the use of atelocollagen in tooth extraction sockets at an early stage at both the morphological and the molecular levels.

\section{Materials and methods}

\section{Animals}

Thirty-six male Sprague-Dawley rats, weighing 230-260 g each (7 to 8-week-old), were used in this study. The animals were fed water and water-kneaded food and were housed in a room with a $12 \mathrm{hr}$ light/dark cycle. They were acclimatized for 1 week before the experiments. This study protocol was used in accordance with the "Principles of Laboratory Animal Care" and relevant national laws.

\section{Experimental design}

After general anesthesia with sodium thiopental, each rat 
had the right incisor extracted with a modified knife and forceps. After astriction with gauze, a piece of atelocollagen sponge (TeruPlug ${ }^{\circledR}$, Olympus Termo Biomaterials, Corp. Tokyo, Japan) was put into the tooth extraction socket of 18 of the rats. As the control group, the other 18 rats were used without atelocollagen sponges.

For histological observation and analysis, at 1, 2, and 3 weeks after the surgery six rats in each group were sacrificed under general sodium thiopental anesthesia and were perfused intracardially with $4 \%$ paraformaldehyde in $0.2 \mathrm{M}$ phosphate buffer ( $\mathrm{pH}$ 7.3). The maxillae were then removed and were stored in the same fixative at $4^{\circ} \mathrm{C}$ for 2 days. Each specimen was demineralized in $10 \%$ formic acid for 5 days and was then rinsed in $0.2 \mathrm{M}$ phosphate buffered saline (PBS), dehydrated with graded ethanols, and embedded in paraffin. Sections were cut at a mesio-distal sagittal plane at a thickness of approximately $5 \mu \mathrm{m}$ and were collected on glass slides. The sections were stained with hematoxylin and eosin (HE) and were then observed by microscopy. For immunohistochemical staining, paraffin sections were deparaffinized with xylene and were incubated in $3 \%$ hydrogen peroxide with methanol for $13 \mathrm{~min}$ at room temperature to block endogenous peroxidase activity. For antigen retrieval, sections were treated with $3 \%$ bovine serum albumin (BSA) or $10 \%$ goat serum for $30 \mathrm{~min}$ at room temperature. The sections were incubated at room temperature for $60 \mathrm{~min}$ with anti-Ki-67, which reacts with proliferating cells (diluted at 1:100; rabbit polyclonal to Ki67 ab66155, Abcam plc, Cambridge, UK), and then were incubated with a biotinylated secondary antibody: Histofine Simple-stain MAX-PO ${ }^{\circledast}$ (Nichirei Corporation, Tokyo, Japan) for $30 \mathrm{~min}$ at room temperature. Thereafter the sections were rinsed with PBS and were stained with Histofine Simple-stain $\mathrm{DAB}^{\circledR}$ (Nichirei), counterstained
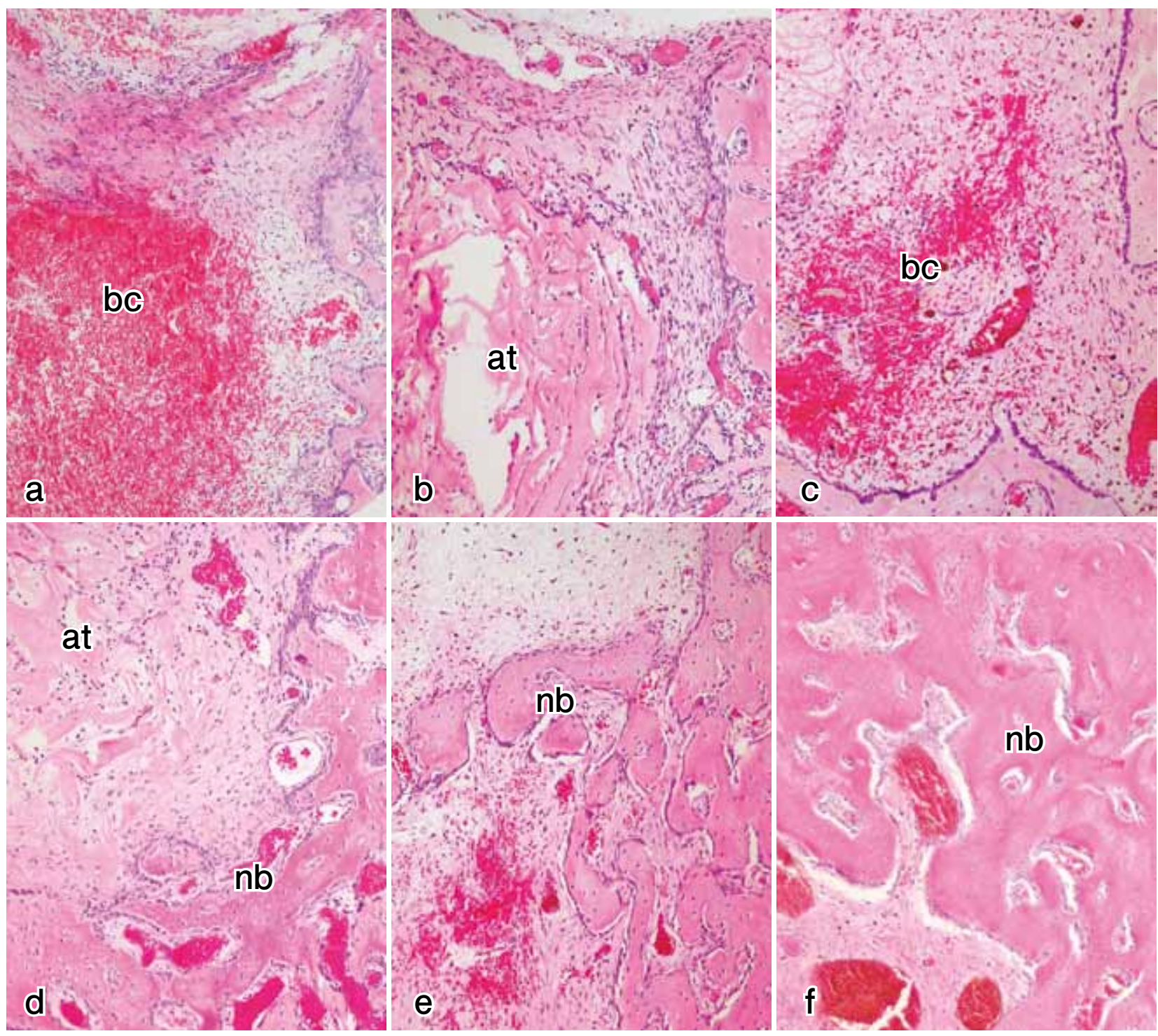

Fig. 1. Histological findings of tooth extraction sockets. Hematoxylin and eosin (HE) stain. (a-c) Control group; (d-f) experimental group with atelocollagen; (a, d) one week after surgery; (b, e) week 2; (c, f) week 3. × 40. nb, new bone; bc, blood clot; at, atelocollagen. Tooth extraction sockets were largely occupied with blood clots at week 1 in the control group (a), while in the experimental group the presence of atelocollagen suppressed the space of blood clots (d). At week 2, new bone formation was clear in both groups $(b, e)$, but a little blood clot in the control (b) and atelocollagen in the experimental group could be observed (e). At week 3, there was a lot of new bone formation in both groups (c, f). 

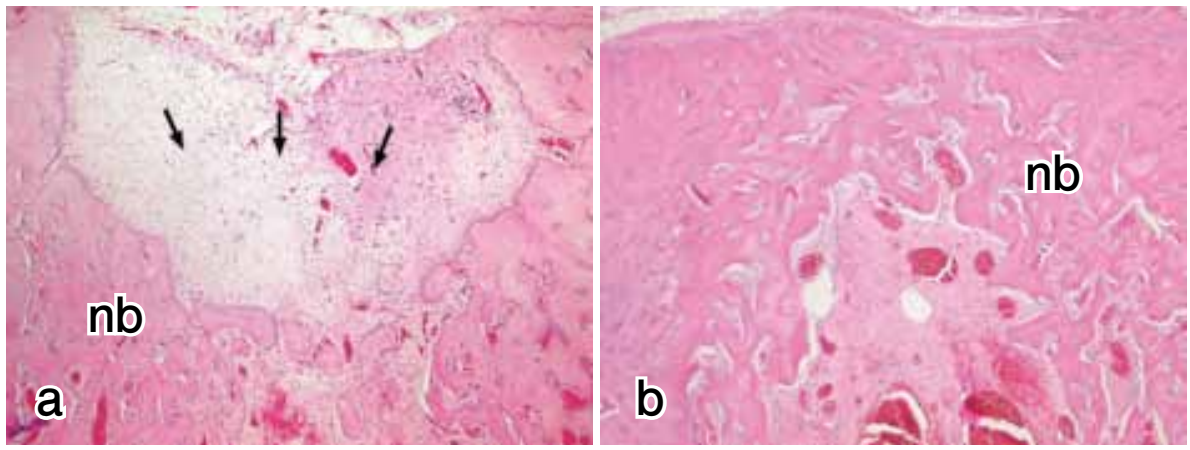

Fig. 2. Histological findings of tooth extraction sockets at week 3 . (a) Control group, (b) experimental group with atelocollagen HE stain, $\times 40$. nb, new bone; arrows, non-specific fibrous connective tissue. In the control, nonspecific fibrous connective tissue invaded the tooth extraction socket at the ostial area, and inhibited to make new bone formation at the alveolar bone ridge area of the socket (a). In the experimental group, however, the orifice of extraction sockets was closed with newly-formed bone trabeculae (b).

with hematoxylin and observed using light microscopy.

Morphometric analysis was carried out at the area of tooth extraction socket from alveolar bone ridge to depth of approximately $2.0 \mathrm{~mm}$. The ratios of blood clot, of atelocollagen, of newly-formed bone and of blood vessel were analyzed using Image J (ver. 1.42, NIH, Bethesda, MD, USA), a software for image analysis. The ratios of Ki-67 positive cells in the specimens were calculated by the following formula: (the number of Ki-67 positive cells/the total number of cells) $\times 100$.

\section{Quantitative RT-PCR}

Total RNAs were extracted using the acid guanidium thiocyanate/phenol-chloroform method as follows: at 1 week after the surgery, tissues in the extraction sockets of depth of approximately $2.0 \mathrm{~mm}$ from the ridge were removed and homogenized in $1 \mathrm{ml}$ TRIsol Reagent (Invitrogen Corporation, Carlsbad, CA, USA), after which each solution was transferred to a $1.5 \mathrm{ml}$ tube containing chloroform and was mixed. Each tube was centrifuged at $14,000 \mathrm{rpm}$ at $4^{\circ} \mathrm{C}$ for $20 \mathrm{~min}$, after which each supernatant was placed in a $1.5 \mathrm{ml}$ tube containing $250 \mu \mathrm{l} 100 \%$ isopropanol (1/4th the amount of TRIsol Reagent) at $-80^{\circ} \mathrm{C}$ for one hr. After centrifugation at 13,200 rpm for $20 \mathrm{~min}$ at $4^{\circ} \mathrm{C}$, the supernatants were discarded and the remaining

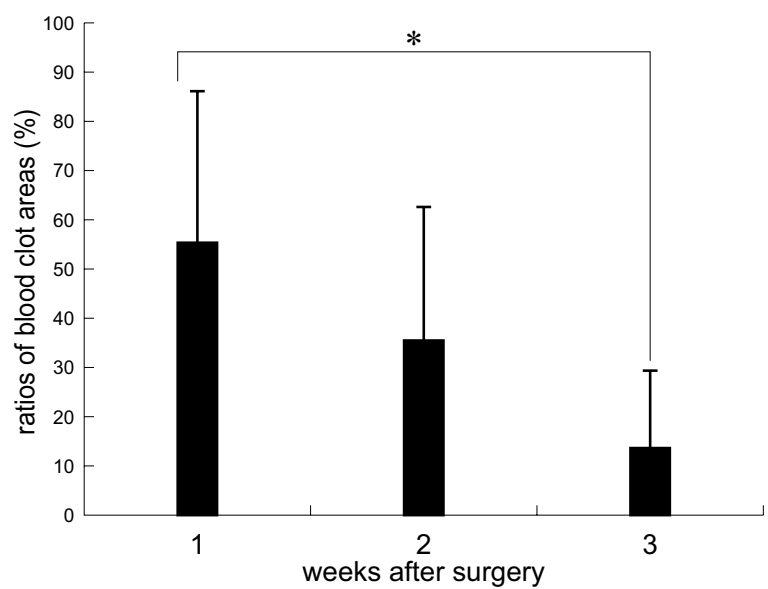

Fig. 3. Morphometric analysis of blood clot areas in the tooth extraction sockets in Figs. 1a, 1c, and 1e. The blood clot area decreased with time after surgery. It was significantly wider at week 1 than at week $3(P<0.05)$. total RNA pellets were washed with $70 \%$ cold ethanol, and then dissolved in $50 \mu \mathrm{l}$ RNAase-free (DEPC-treated) water. Total RNAs were reverse transcribed and amplified in $20 \mu \mathrm{l}$ volumes using a Reverse Transcription Kit (Quanti Tect, Qiagen Inc., Hilden, Germany) containing RNA PCR Buffer, $2 \mathrm{U} / \mu \mathrm{l}$ RNAase inhibitor, $0.25 \mathrm{U} / \mu \mathrm{M}$ reverse transcriptase, $0.125 \mu \mathrm{M}$ oligo dt-adaptor primer, $5 \mathrm{mM}$ $\mathrm{MgCl}_{2}$ and RNAase-free water. RT-PCR products were analyzed by quantitative real-time RT-PCR in TaqMan Gene Expression Assays (Applied Biosystems, Life Technologies Corporation, Carlsbad, CA, USA) for the target genes: type I collagen (Custom Taqman(R) Gene Expression Assay Service, Forward: GGTGCCCCTGGTGACAAG, Reverse: GGGCCAGCAGGACCA, Probe: CTGGGACCAGTTTCAC, Applied Biosystems), and osteocalcin (OCN, Rn01455285_g1, Applied Biosystems). The TaqMan Endogenous Control for the target gene $\beta$-actin (TaqMan Endogenous Control 4352340E, Applied Biosystems) was used as a control. All PCR reactions were performed using a real time PCR 7500 fast system. Gene expression quantitation using TaqMan Gene Expression Assays was performed as the second step in a two-step RT-PCR. Assays were done in $20 \mu \mathrm{L}$ singleplex reactions containing TaqMan Fast Universal PCR Master Mix, TaqMan Gene Expression Assays, distilled water and cDNA, according to the

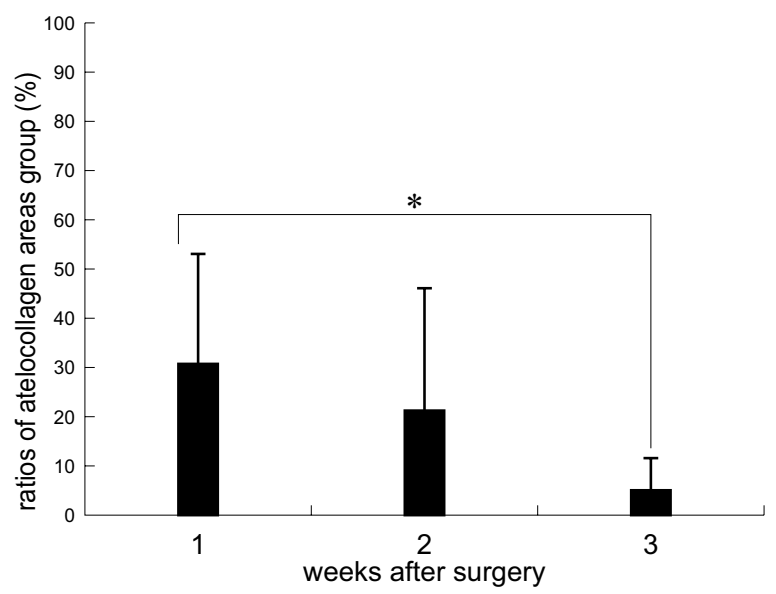

Fig. 4. Morphometric analysis of atelocollagen areas in the tooth extraction socket in experimental group in Figs. $1 \mathrm{~b}, 1 \mathrm{~d}$, and 1f. The area of atelocollagen at week 1 , estimated from the picture of Fig. 1, was significantly wider than at week $3(P<0.05)$. 


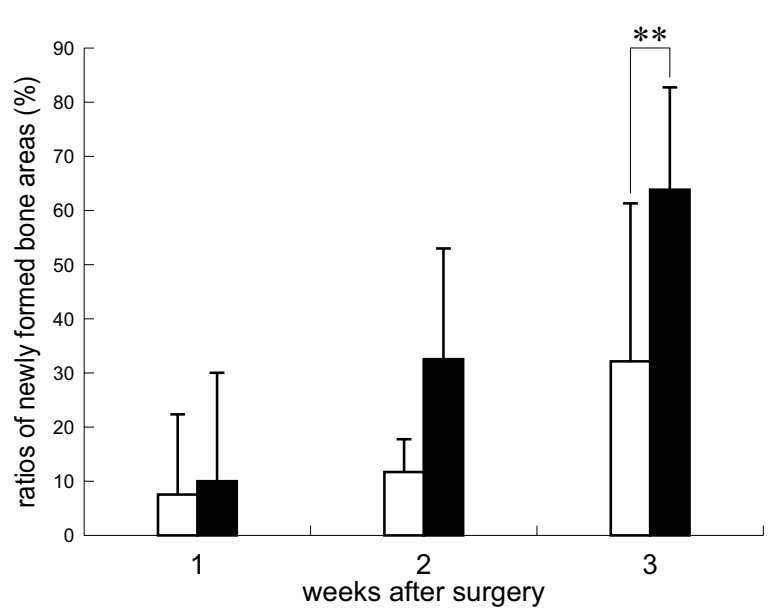

Fig. 5. Morphometric analysis of newly-formed bone areas in the tooth extraction socket with or without atelocollagen. $\square$, control group; $\square$, experimental group. The ratio of newly-formed bone in the experimental group was significantly higher than that in the control group $(P<0.01)$.

manufacturer's instructions (Applied Biosystems). Reaction conditions consisted of a primary denaturation at $95^{\circ} \mathrm{C}$ for $20 \mathrm{sec}$, then cycling for 40 cycles of $95^{\circ} \mathrm{C}$ for $3 \mathrm{sec}$ and $62^{\circ} \mathrm{C}$ for $30 \mathrm{sec}$. Quantitative RT-PCR analyses were reproduced three times.

\section{Statistical analysis}

Data were analyzed via one-way ANOVA and were compared by Scheffe's test.

\section{Results}

\section{Histological observation}

$\mathrm{H}-\mathrm{E}$ staining revealed that a small amount of new bone was formed beside the alveolar bone in the control and experimental groups 1 week after surgery (Fig. 1). A blood clot had filled the sockets in the control group but atelocollagen could be seen in the experimental group. A small blood clot in the control and some atelocollagen remained at 2 weeks after the surgery. At 3 weeks after the surgery, the blood clot in the control group and the atelocollagen in the experimental group had disappeared, and newly-formed bone filled the sockets. Furthermore, non-specific fibrous connective tissue invaded the sockets from outside in the control group, but new bone formation could be seen up to the level of the alveolar bone ridge in the experimental group (Fig. 2).

\section{Morphometric analyses}

Blood clots in the experimental group could not be observed at all time periods. The ratios of blood clot areas among the socket space in control group decreased in a time-dependant manner (Fig. 3). The ratio of atelocollagen areas in the experimental group decreased in a timedependant manner (Fig. 4). The ratios of newly-formed bone trabecula areas in both groups increased day by day, and those in the experimental group at 2 and 3 days were significantly higher than those in the control group $(P<0.01)$

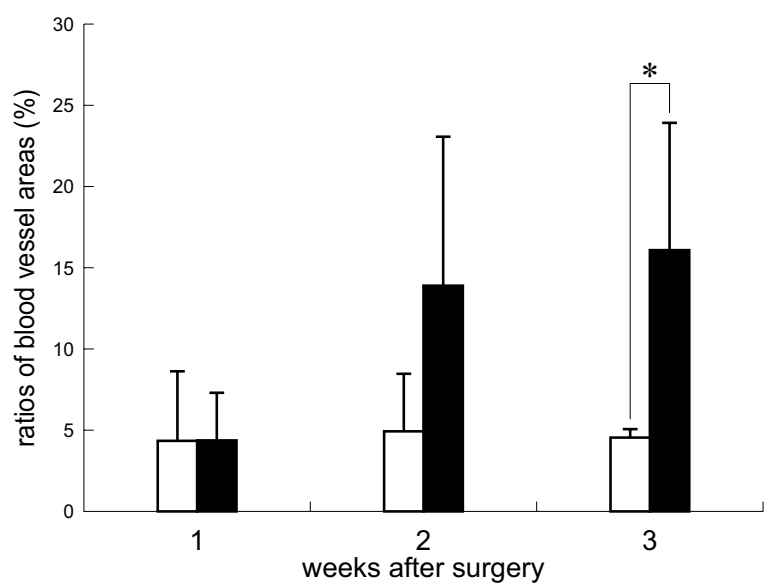

Fig. 6. Morphometric analysis of blood vessel areas in the tooth extraction socket with or without atelocollagen. $\square$, control group; $\square$, experimental group. The area of blood vessel in the experimental group at 3 weeks was significantly higher than that in the control group $(P<0.01)$.

(Fig. 5). The ratio of blood vessels in the experimental group at 3 weeks was higher than in the control group $(P<0.01)$ (Fig. 6).

\section{Immunohistochemisatry for cell proliferation}

Ki-67 positive cells could be seen in the sockets of both the control and the experimental groups (data not shown). In addition, Ki-67 positive cells could be seen in the space among atelocollagen fibers. There was no significant difference between them, but the ratio in the experimental group was tended to higher than that in the control group. Furthermore, there was no significant difference between the control and the experimental groups at any time period.

\section{mRNA expression levels}

Expression of type I collagen mRNA in the experimental group at 1 week after the surgery tended to be higher than in the control group (Fig. 7), but at 2 weeks after the surgery there was no significant difference between the experimental and the control groups (data not shown). On the other hand, expression of OCN mRNA was not significantly different between the experimental and the control groups at 1 or 2 weeks after the surgery.

\section{Discussion}

Atelocollagen is used for many kinds of medical therapies, such as a scaffold for medicine (1-2) or stem cells (2, 5-6), hemostasis, and so on. For dental treatment, atelocollagen is also used for hemostasis and to maintain the alveolar bone ridge after tooth extraction (7-8). Although atelocollagen is clinically used for hemostasis after tooth extraction, in this study it could be seen in the sockets instead of the blood clot 1 week after the surgery. This means that atelocollagen plays a role in hemostasis of the tissue remodeling process in tooth extraction sockets. The present experimental results are schematically summarized in Fig. 8. Atelocollagen has been used to maintain the shape 


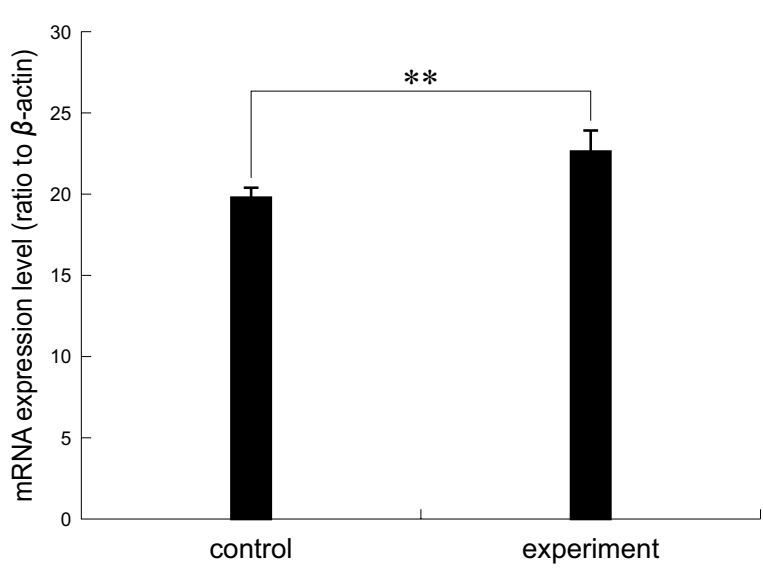

Fig. 7. Comparative type I collagen mRNA expression levels in the tooth extraction socket with or without atelocollagen at week 1 . The mRNA expression in the experimental group was higher than in the control group $(P<0.01)$.

of the alveolar bone ridge, and also in this study we demonstrated that atelocollagen functioned as a spacemaintaining factor. In the presence of atelocollagen, the sockets were filled with newly-formed bone trabeculae, while in the controls they were invaded by non-specific fibrous connective tissue which invaded from the outside.

$\mathrm{Ki}-67$ is related to cell proliferation and is used as the marker of cell activity (9-10). In this study, Ki-67 positive cells could be seen in the tooth extraction sockets in both the control and the experimental groups. Ki-67 positive ratio in experimental group at 1 week tended to be higher than that in the control group, but there was no significant difference between the control and the experimental groups. This means that although wound healing depends mainly on cell proliferation, atelocollagen does not accelerate cell proliferation in the tooth extraction sockets.

Expression of type I collagen mRNA in the experimental group at 1 week after the surgery had a tendency to be higher than in the control group, but at 2 weeks after the surgery there was no significant difference between the experimental and the control groups. It is known that type I collagen plays a role in an extremely early stage of bone formation (11), so these results reveal that atelocollagen causes the cells in the tooth extraction sockets to secrete the extracellular matrix. However, the expression of OCN mRNA was not significantly different between the experimental and the control groups at 1 or 2 weeks after the surgery. $\mathrm{OCN}$ is a noncollagenous protein, and is secreted at the beginning stage for calcification of the bone (12). Since the expression of OCN mRNA was not significantly different between the experimental and the control groups, atelocollagen does not seem to have a role in osteogenic induction, but is involved in extracellular matrix production. These results reveal that atelocollagen plays a role not only in maintaining the spacing during tooth wound repair but also in prompting the production of the extracellular matrix.

In this study, the ratio of newly-formed bone in the experimental group was significantly higher than that in the control group, but the OCN mRNA expression showed no significant difference between both groups. Furthermore,

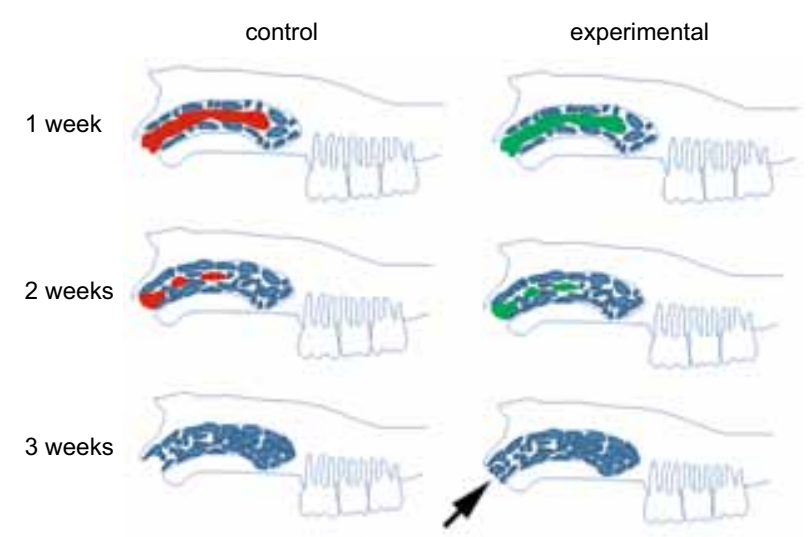

Fig. 8. Schematic summary of the experiment on atelocollagen effects in wound healing of rat tooth extraction sockets. , newly-formed bone; , blood clot; , atelocollagen. At week 1, tooth extraction sockets were occupied with blood clots in the control group, and the presence of atelocollagen suppressed the space of blood clots in the experimental group. At week 2, new bone formation was observed in both groups, but a little blood clot in the control and atelocollagen in the experimental group could be still observed. At week 3 , there was abundant new bone formation in both groups. Note that the orifice of the extraction sockets was closed with newly-formed bone (arrows). Atelocollagen has been used to maintain the shape of the alveolar bone ridge, and also in this study we demonstrated that atelocollagen functioned as a space-maintaining factor. In the presence of atelocollagen, the sockets were filled with newlyformed bone trabeculae, while in the control they were invaded by non-specific fibrous connective tissue invaded from the outside.

the ratio of blood vessels in the experimental group at 3 weeks was significantly higher than that in the control group. Rich blood flow plays a role in the healing of bone. This study indicated that atelocollagen has an advantage for new bone formation in tooth extraction sockets. Further Ki-67 positive cells can be seen in the space among atelocollagen fibers.

In conclusion, atelocollagen plays a role in maintaining space and in producing collagen as an extracellular matrix, but does not accelerate the cell proliferation or osteoinduction.

\section{Acknowledgments}

We would like to thank Ms. Saori Takano and members of the Department of Clinical Pathophysiology, Tokyo Dental College, for their technical assistance. This research was in part supported by an Oral Health Science Center Grant HRC7 from the Tokyo Dental College, and by a "High-Tech Research Center" Project for Private Universities: matching fund subsidy from MEXT (Ministry of Education, Culture, Sports, Science and Technology) of Japan, 2006-2010, and 2007-2010 (No. 19592414).

\section{References}

1. Nakanishi A, Hakamada A, Isoda K, Mizutani H. Atelocollagen sponge and recombinant basic fibroblast growth factor combination therapy for resistant wounds 
with deep cavities. J Dermatol 2005; 32: 376-80.

2. Minabe M, Kodama T, Hori T, Watanabe Y. Effects of atelocollagen on the wound healing reaction following palatal gingivectomy in rats. J Periodontal Res 1989; 24: 178-85.

3. DeLustro F, Condell RA, Nguyen MA, McPherson JM. A comparative study of the biologic and immunologic response to medical devices derived from dermal collagen. J Biomed Mater Res 1986; 20: 109-20.

4. Maehara H, Sotome S, Yoshii T, Torigoe I, Kawasaki Y, Sugata Y, Yuasa M, Hirano M, Mochizuki N, Kikuchi M, Shinomiya K, Okawa A. Repair of large osteochondral defects in rabbits using porous hydroxyapatite/collagen (HAp/Col) and fibroblast growth factor-2 (FGF-2). $J$ Orthop Res 2009; 28: 677-86.

5. Ohno S, Hirano S, Tateya I, Kanemaru S, Umeda H, Suehiro A, Kitani Y, Kishimoto Y, Kojima T, Nakamura T, Ito J. Atelocollagen sponge as a stem cell implantation scaffold for the treatment of scarred vocal folds. Ann Otol Rhinol Laryngol 2009; 118: 805-10.

6. Nambu M, Kishimoto S, Nakamura S, Mizuno $H$, Yanagibayashi S, Yamamoto N, Azuma R, Nakamura S, Kiyosawa T, Ishihara M, Kanatani Y. Accelerated wound healing in healing-impaired $\mathrm{db} / \mathrm{db}$ mice by autologous adipose tissue-derived stromal cells combined with atelocollagen matrix. Ann Plast Surg 2009; 62: 317-21.

7. Iibuchi S, Matsui K, Kawai T, Sasaki K, Suzuki O,
Kamakura S, Echigo S. Octacalcium phosphate (OCP) collagen composites enhance bone healing in a dog tooth extraction socket model. Int J Oral Maxillofac Surg 2010; 39: 161-8.

8. Yamaoka H, Tanaka Y, Nishizawa S, Asawa Y, Takato T, Hoshi K. The application of atelocollagen gel in combination with porous scaffolds for cartilage tissue engineering and its suitable conditions. J Biomed Mater Res A 2009; 93: 123-32.

9. Seoane J, Pita-Fernández S, Gómez I, Vazquez I, LópezCedrún JL, De Agustin D, Varela-Centelles P. Proliferative activity and diagnostic delay in oral cancer. Head Neck 2010; 32:, online published, doi: 10.1002/hed.21338.

10. El-Zammar O, Rosenbaum P, Katzenstein AL. Proliferative activity in fibrosing lung diseases: a comparative study of Ki-67 immunoreactivity in diffuse alveolar damage, bronchiolitis obliterans-organizing pneumonia, and usual interstitial pneumonia. Hum Pathol 2009; 40: 1182-8.

11. Canalis E. Effect of glucocorticoids on type I collagen synthesis, alkaline phosphatase activity, and deoxyribonucleic acid content in cultured rat calvariae. Endocrinology 1983; 112: 931-9.

12. Akahane $M$, Shigematsu $H$, Tadokoro $M$, Ueha $T$, Matsumoto T, Tohma Y, Kido A, Imamura T, Tanaka Y. Scaffold-free cell sheet injection results in bone formation. J Tissue Eng Regen Med 2010; 4:, online published, doi: $10.1002 /$ term. 259 . 